\title{
Measurement of constriction size distributions using three grain-scale methods
}

\author{
T. Shire, C. O’Sullivan \& H. Taylor \\ Imperial College, South Kensington, London, UK \\ W.W. Sim \\ Atkins Ltd, Bristol, UK
}

\begin{abstract}
The grain-scale justification for empirical rules for granular filters has largely been based on simplified models of sphere packings. The development of discrete element modelling (DEM) and micro computed tomography $(\mu \mathrm{CT})$ enables a more scientific appraisal of the void space within granular filter materials. The constrictions or pore throats that govern the filter's performance can now be directly measured. However, definitive partitioning of the void space is not possible for realistic grain packings and so multiple methods, with differing theoretical bases, have been proposed to identify constrictions. This contribution compares three such methods, each of which results in a constriction size distribution (CSD). The methods considered are the triangulation based weighted Delaunay method (Reboul et al. 2010), a contact based method (O'Sullivan et al. 2015) and an image analysis method based on watershed segmentation (Taylor et al. 2015). Each model, along with its relative advantages is introduced. Then CSDs resulting from applying each model to the same virtual filter samples created using DEM are presented. It is shown that there is reasonable agreement despite the different basis of each approach.

A consideration of empirical filter rules is carried out by normalising the full CSDs by the characteristic diameters typi-cally used to represent the retention capacity of granular filters in design. It is shown that similar CSD curves are ob-tained for different particle size distributions (PSDs) when curves are normalised by characteristic diameters, irrespec-tive of the method used to identify the constrictions. This gives fundamental support to filter rules using characteristic particle diameters to represent the filtration capability of granular filters.
\end{abstract}

\section{INTRODUCTION}

Granular filters retain base material within the narrow constrictions of the void network. Void space is three-dimensional and continuous throughout a granular filter, making the identification of the narrow constrictions which form the boundary between voids a difficult and somewhat subjective task. This paper presents three methods which can be used to identify individual constrictions, and therefore a constriction size distribution (CSD) within a granular material. The three methods are outlined and then applied to the same virtual granular filter samples, generated using the grain scale discrete element modelling (DEM). The results compare the three methods, following which the effect of the coefficient of uniformity $\left(\mathrm{C}_{\mathrm{u}}\right)$ on CSD is studied. Finally, a fundamental explanation is sought for Terzaghi's classic filter rule $D_{85 \mathrm{~B}}>\mathrm{D}_{15 \mathrm{~F}} / 4$, where $\mathrm{D}_{15 \mathrm{~F}}$ is the filter diameter for which $15 \%$ of material is smaller and $\mathrm{D}_{85 \mathrm{~B}}$ is the base diameter for which $85 \%$ of material is smaller (Terzaghi \& Peck, 1948), by comparing CSDs normalized by $\mathrm{D}_{15 \mathrm{~F}}$ for different filters.

\subsection{Background}

Granular filters are typically designed using filter rules which compare a characteristic filter particle size with the particle size of the base material which they must retain (e.g. Sherard \& Dunnigan (1989); ICOLD (2015)). Since Terzaghi's original filter rule a link has been made between the characteristic filter particle diameter and the size of the constrictions within the voids, i.e. the $\mathrm{D}_{15 \mathrm{~F}} / 4$ term in Terzaghi's equation represents the constriction diameter (Fannin, 2008).

Kenney et al. (1985) carried out extensive filter testing using filters with $\mathrm{Cu}=1.2$ to 12 to determine the 'controlling constriction size' which they defined as the smallest constriction which a base particle is likely to encounter on a given flow path through the filter. They found that across this range of filters both $\mathrm{D}_{5 \mathrm{~F}}$ and $\mathrm{D}_{15 \mathrm{~F}}$ gave good estimates of the controlling constriction. Shire \& O'Sullivan (2015) determined the CSDs for virtual granular filters with $\mathrm{C}_{\mathrm{u}}=1.2$ to 6 and varying relative density using the Weighted Delaunay method (Reboul et al., 2010). Shire \& O'Sullivan (2015) found that the CSD 
curves had similar shapes, and when normalized by $\mathrm{D}_{5 \mathrm{~F}}$ or $\mathrm{D}_{15 \mathrm{~F}}$ the CSD curves had similar values. This can be considered a scientific justification for using $\mathrm{D}_{5 \mathrm{~F}}$ or $\mathrm{D}_{15 \mathrm{~F}}$ as characteristic diameters for filter design, echoing the experimental findings of Kenney et al. (1985).

\section{METHODOLOGY}

\subsection{Measurement of constrictions}

Modern techniques such as DEM (O'Sullivan, 2011) and micro-computed tomography $(\mu \mathrm{CT})$ (Ketcham \& Carlson, 2001) allow the full CSD of a granular filter to be directly measured. Three methods for identifying constrictions within the DEM samples were used. These are the Weighted Delaunay Method (Reboul et al., 2010), the Contact Based Method (O'Sullivan et al., 2015) and the Watershed Method (Taylor et al., 2015). Each method has two stages: (i) identification of individual voids within the continuous, 3D void space; (ii) identification and quantification of constrictions at the boundaries between these voids.

\subsection{Weighted Delaunay Method}

The Weighted Delaunay tessellation-based method was originally proposed by Al-Raoush et al. (2003) and Reboul et al (2010) and is applicable to spherical particles. A weighted Delaunay triangulation is carried out on the particle centroids weighted by their diameter, here the Regular Triangulation feature in CGAL (2013) was used. This creates a series of tetrahedral Delaunay cells between particles and the void space within each cell is considered to form a void (Figure 1). Using a single Delaunay cell to define a void leads to over-segmentation of the void space, as shown using a 2D analogy in Figure 2(a). Therefore Delaunay cells are merged using a criterion originally proposed by Al-Raoush et al (2003), which considers the largest spheres that can be inscribed between the particles making up a single Delaunay cell. Merging takes place if the overlap between the inscribed spheres, expressed as a percentage of the smaller particle radius, exceeds a user-defined level, shown in 2D in Figure 2(b). As the selected critical overlap decreases, fewer, larger voids will be created. The selection of the critical overlap is subjective, meaning that no unique CSD can be defined. It should be noted that the separation of a 3D void space into discrete voids will always be somewhat subjective, whatever method is used. Following a parametric study a critical overlap of $50 \%$ of the smaller inscribed sphere radius was selected.

Once individual voids have been identified, constrictions are measured on the Delaunay cell faces on the outside of voids. A constriction is defined as the smallest circle which can be inscribed between the three particles making up vertices or a combination of the vertex particles and any non-vertex particles which overlap the face (Reboul et al, 2010).

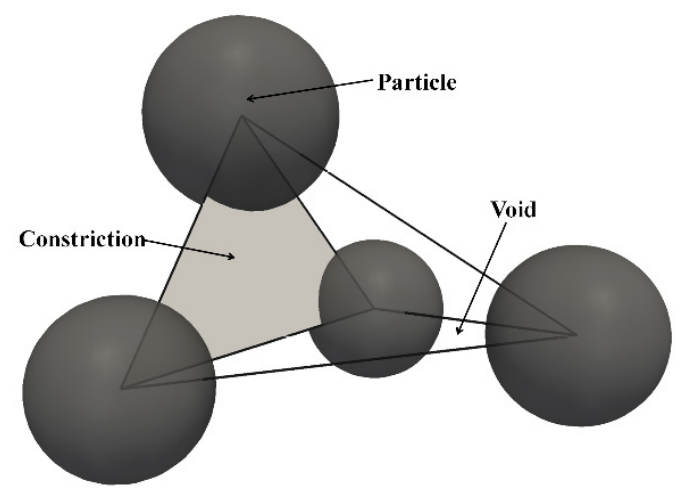

Figure 1. Use of Delaunay tetrahedral cell to define voids and constrictions

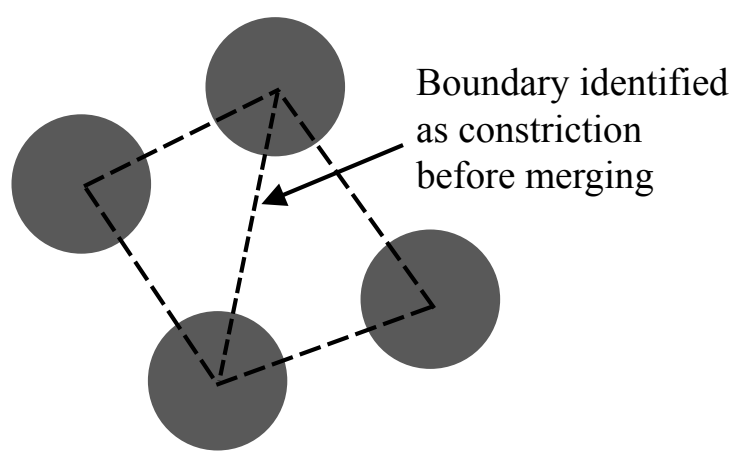

(a)

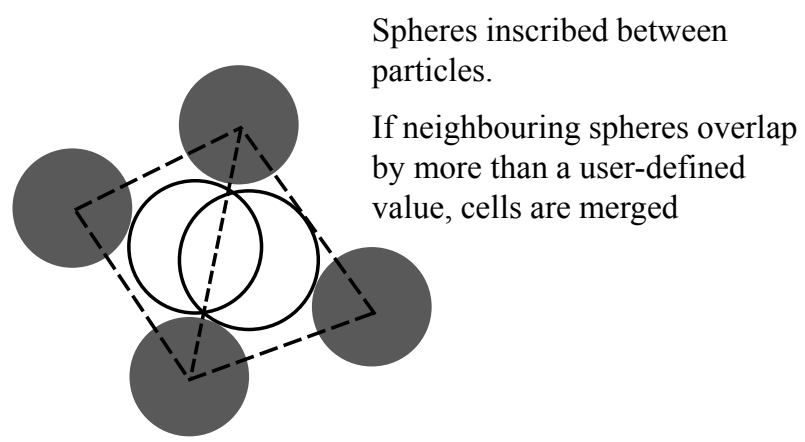

(b)

Figure 2. Merging Delaunay cells to form larger voids

\subsection{Contact Based Method}

The contact based method was proposed by O'Sullivan et al. (2015) and is a 3D extension of a 2D method proposed by Li \& Li (2009). This algorithm is based on a Delaunay triangulation of the contact points between particles, as shown using a 2D analogy in Figure 3(a). The input comprises the particle centroids, particle radii, a list of contacting disks and the contact coordinates.

After the Delaunay triangulation of contact points has been carried out the resulting tetrahedral cells are classified as either solid or void cells. Solid cells are defined as cells for which all four contact points forming the vertices share a common particle. In this case the cell must be predominantly enclosed within the common particle. The remaining cells are classi- 
fied as void cells (tetrahedra in 3D), as shown in 2D in Figure 3(b). The faces of the void cells are then analysed to determine if: (i) they are constrictions linking two voids or (ii) the two void cells which share the face should be merged to form a larger void (Figure 3(c)).

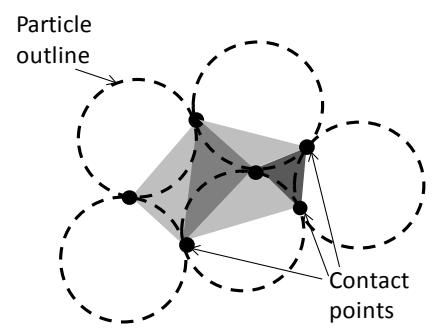

(a)

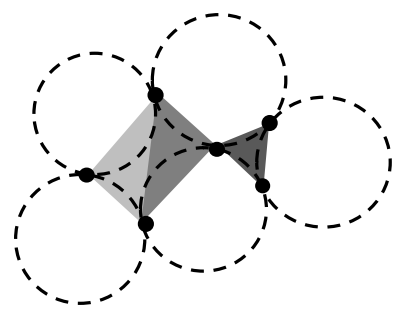

(b)

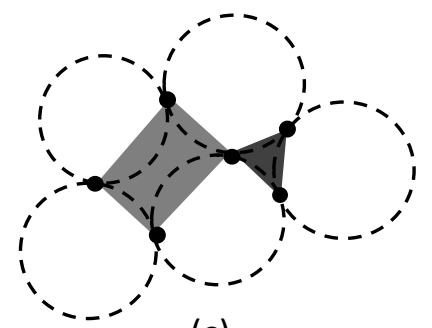

(c)

Figure 3. Contact based method: (a) Delaunay triangulation of contacts; (b) removal of solid cells; (c) merging of void cells

With reference to Figure 4(a) and (b), if the vertices of a cell face consist of contacts between particles forming a closed loop of three or four contacting particles then the face is defined as a constriction between two voids. With reference to Figure 4(c), where the vertices of a cell face are not part of such a closed loop the two cells which share this face are merged to form part of a larger void.

Once the cell faces representing constrictions have been identified the largest circle which can be inscribed on this face is calculated. The diameter of this inscribed circle represents the constriction diameter.

\subsection{Watershed Method}

The Watershed Method was developed by Taylor et al. (2015) to allow the identification of constrictions within $\mu \mathrm{CT}$ images of real granular filters. It takes as an input a voxelised binary image in which voxels (3D pixels) represent either void space or soil particles (Figure 5).

The advantage of using a voxelised image is that any material consisting of void space and solid particles can be analysed. The main disadvantages of the image based technique is that it is memory intensive - a minimum of around 30 voxels per particle diameter is required for accurate identification of constrictions, meaning that the number of particles which could be analysed in an image of $600^{3}$ voxels was fewer than could be used for the Weighted Delaunay or Contact Based methods.

The void space part of the image is partitioned into individual voids using a watershed method (Taylor et al., 2015). A distance map is created which gives the distance that each void voxel is from the nearest solid voxel. This can be considered to be analogous to a map of topographical elevations, with a greater distance from a void being considered a lower elevation. As illustrated in Figure 6, the watershed algorithm then determines the locations of the 'watersheds' on this distance map. These watersheds are considered to be the boundaries between voids. In order to screen out small local variations in the distance map which could produce false void boundaries, the minimum peak-to-trough height (shown by the grey arrow in Figure 6) was set to be $1 / 10^{\text {th }}$ of the minimum particle diameter.

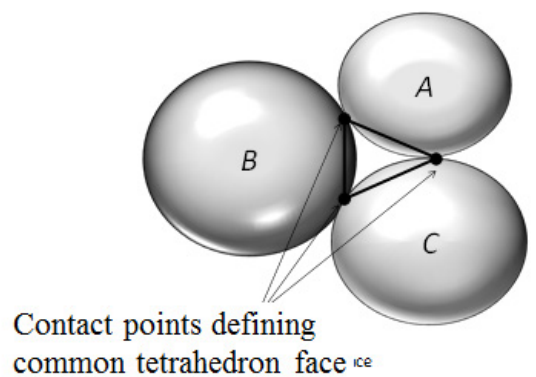

(a)

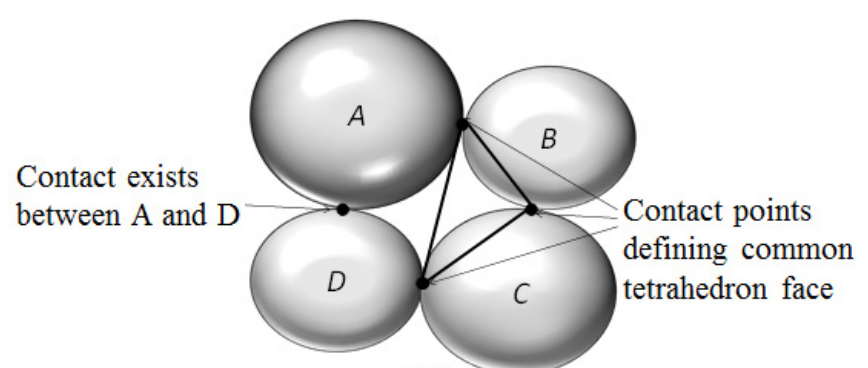

(b)

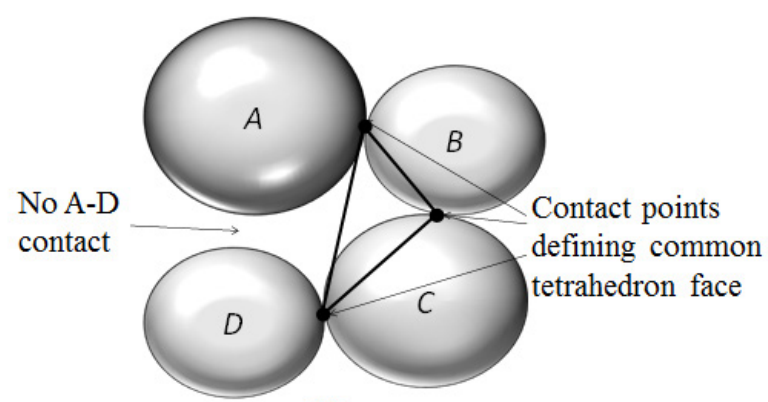

(c)

Figure 4. Identification of constrictions in contact based method: (a) three-particle constriction; (b) four-particle constriction; (c) face connecting two void cells which are part of a single, larger void (O’Sullivan et al., 2015)

Unlike the Contact and Weighted Delaunay Methods, constriction boundaries identified by the Watershed Method are not planes, but are irregular surfaces, as shown in Figure 7. On these irregular surfaces the constriction diameters are defined as the 
local maxima of the distance map on the constriction surface. This leads to the identification of many individual constrictions on the constriction surface (Figure 7(a)). Taylor et al. (2015) outline a four step procedure to remove the erroneous local maxima so that only meaningful constrictions are identified. For large, irregular constriction surfaces more than one valid constriction can be identified (Figure 7(b)).

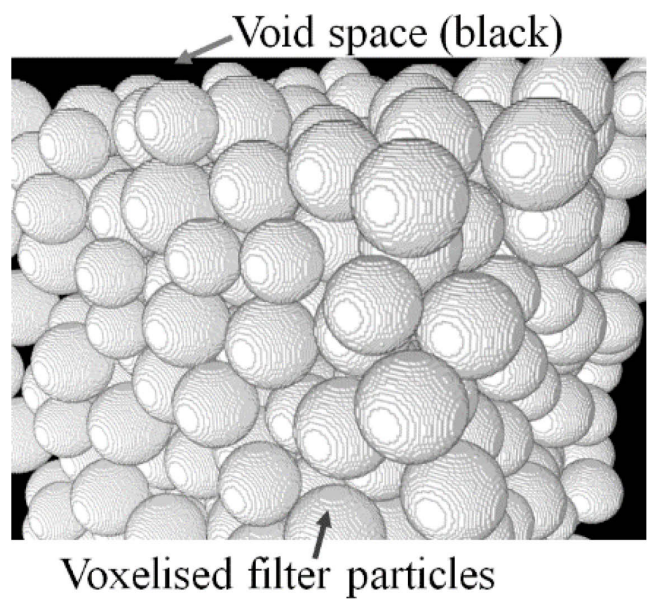

Figure 5. Voxelised image with voxels representing either solid space (white) or void space (black).

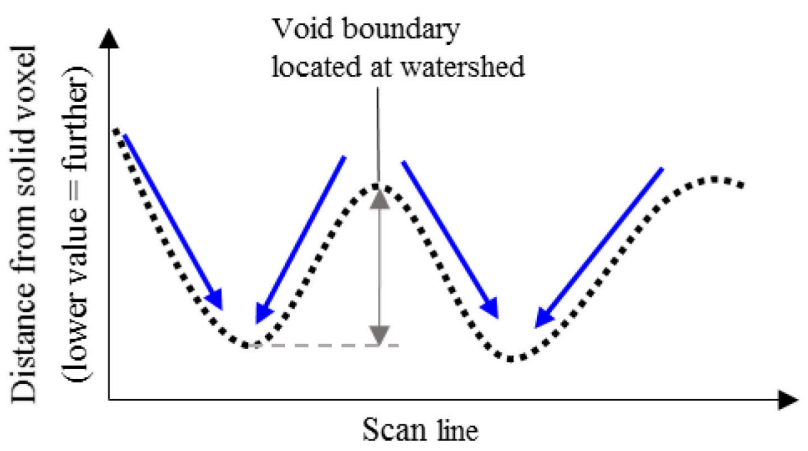

Figure 6. Conceptual diagram showing watershed algorithm

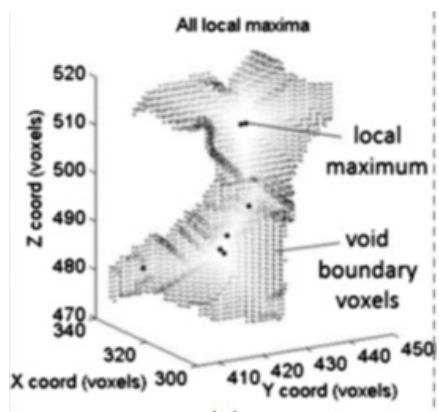

(a)

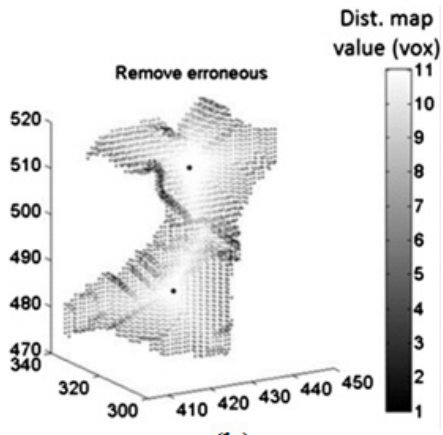

(b)
Figure 7. Constriction surfaces (Taylor et al., 2015). (a) All local maxima; (b) erroneous maxima removed to leave only true constrictions

\section{GENERATION OF FILTER SAMPLES}

Virtual granular filters were generated using the DEM code granular LAMMPS (Plimpton 1995). The filters have linearly graded PSDs with $C_{u}=1.2$ to 6 , as shown in Figure 8. Each filter was generated with periodic boundaries and particles initially randomly placed in the model domain. The filters were then isotropically compressed to a mean normal stress $\mathrm{p}^{\prime}$ $=\left(\sigma_{1}^{\prime}+\sigma_{2}^{\prime}+\sigma_{3}^{\prime}\right) / 3=50 \mathrm{kPa}$ where $\sigma_{1}^{\prime}, \sigma_{2}^{\prime}, \sigma_{3}^{\prime}$ are the three principal stresses. An inter-particle friction coefficient of $\mu=0.3$ was used, which was selected to match the measured value of glass beads and allowed a relatively loose sample to be created. The number of particles used in each simulation is shown in Table 1. A subset of each sample was used for the more memory intensive Watershed Method. The Watershed algorithm was applied only to the samples with $\mathrm{C}_{\mathrm{u}}=1.5,3$ and 4.5 .

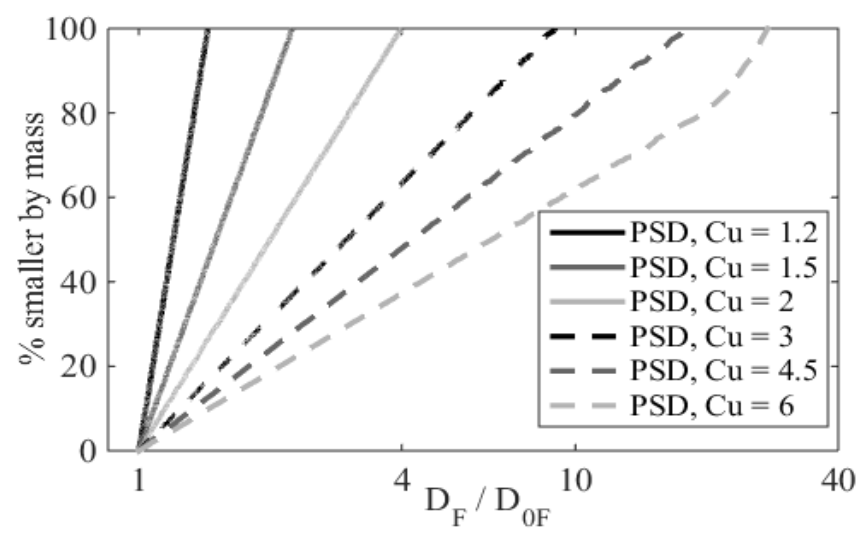

Figure 8. Particle size distributions of filters analysed

Table 1: Number of particles used to calculate constrictions

\begin{tabular}{|c|c|c|c|}
\hline \multirow{2}{*}{$\begin{array}{l}\text { Coefficient } \\
\text { of uni- } \\
\text { formity }\end{array}$} & \multirow{2}{*}{$\begin{array}{l}\text { Void } \\
\text { ratio }\end{array}$} & \multicolumn{2}{|c|}{ Number of particles analysed } \\
\hline & & $\begin{array}{l}\text { Weighted Delaunay } \\
\text { and Contact }\end{array}$ & Watershed \\
\hline 1.2 & 0.714 & 8262 & N/A \\
\hline 1.5 & 0.658 & 9313 & 2476 \\
\hline 2 & 0.555 & 12115 & N/A \\
\hline 3 & 0.455 & 22600 & 10189 \\
\hline 4.5 & 0.384 & 44821 & 8567 \\
\hline 6 & 0.314 & 59183 & $\mathrm{~N} / \mathrm{A}$ \\
\hline
\end{tabular}

\section{RESULTS}

\subsection{Comparison of CSDs generated with different methods}

Figure 9(a-c) shows the constriction size distributions generated using each of the three methods with filters of $C_{u}=1.5,3$ and 4.5 respectively. In each case the constriction diameters, $D_{c}$ are normalized by the smallest filter particle $\mathrm{D}_{0 \mathrm{~F}}$. For the Weighted Delaunay Method the variation of the CSD with merging overlap is also presented. 
It can be seen in Figure 9 that the methods generate broadly comparable CSDs with slightly better agreement between the CSDs for $\mathrm{Cu}=3$ and 4.5 than for $\mathrm{Cu}=1.5$. Each method results in a minimum constriction size which approximately matches the analytical minimum for spherical particles $\mathrm{D}_{\mathrm{c} \text {,min }}$ $\approx 0.155 \mathrm{D}_{0 \mathrm{~F}}$. The Weighted Delaunay CSD varies depending on the overlap chosen for void merging the variation in CSD resulting from a $0 \%$ to $100 \%$ change in overlap is of a similar magnitude to the variation between the three methods.

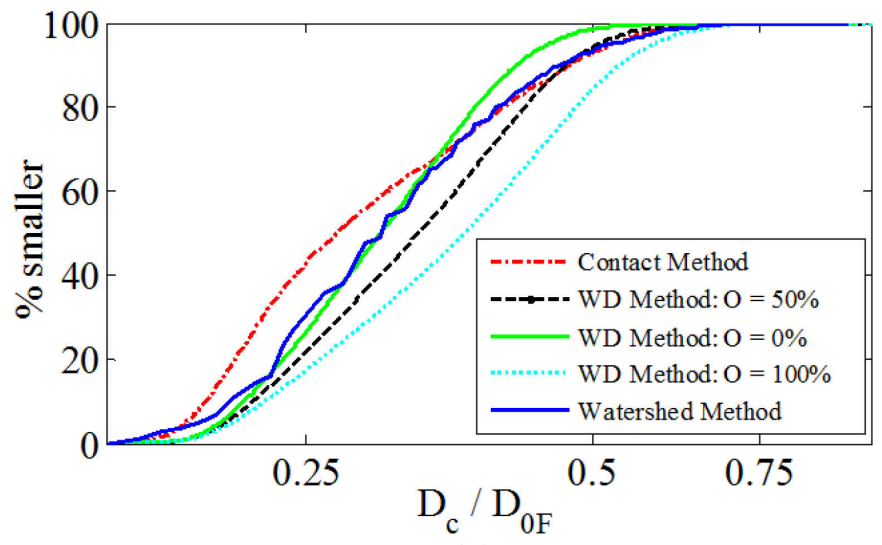

(a)

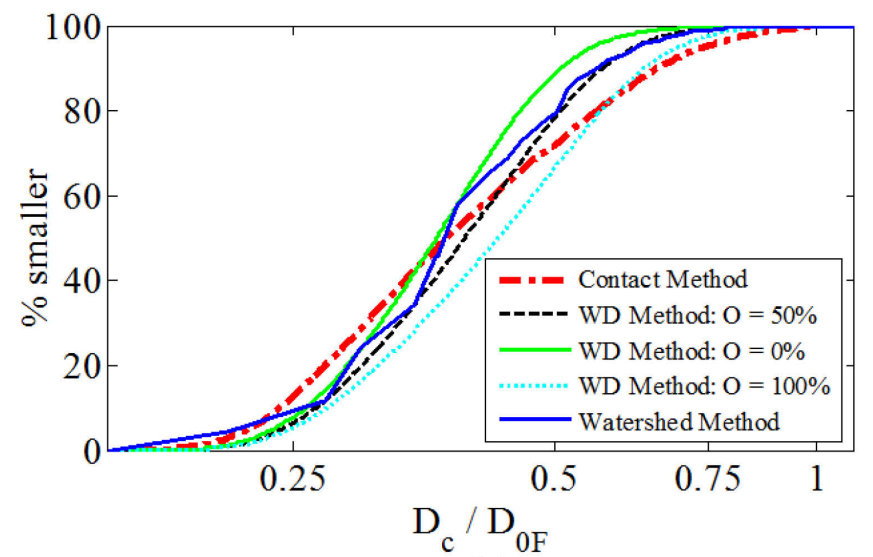

(b)

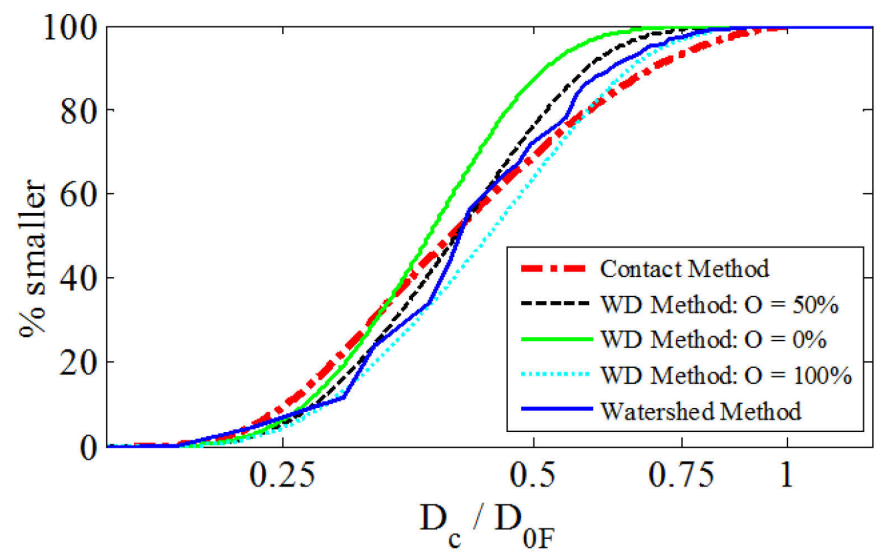

(c)

Figure 9. Comparison of normalized constriction size distributions using different methods: (a) Filter $\mathrm{C}_{\mathrm{u}}=1.5$; (b) $\mathrm{C}_{\mathrm{u}}=3$; $\mathrm{C}_{\mathrm{u}}$ $=4.5$. Overlap $(\mathrm{O})$ of $0,50 \& 100 \%$ presented for WD Method
The fact that three constriction algorithms, each of which has a logical basis, generate different CSDs again highlights the fact that there is no unique CSD for a given granular filter.

\subsection{Variation of CSD with coefficient of uniformity}

Figures 10 to 12 present the CSDs for all filters analysed, which had coefficient of uniformity $\left(C_{u}\right)$ values of $\mathrm{C}_{\mathrm{u}}=1.2$ to 6 for each of the three methods used. In each case the constriction diameters are normalized by the smallest filter particle $\left(\mathrm{D}_{0 \mathrm{~F}}\right)$ and the commonly characteristic filter diameter $D_{155}$. Where $\mathrm{D}_{15 \mathrm{~F}}$ is used the range of median constriction diameters are also given on the figure.

With reference to Figures 10(a), 11(a) and 12(a), the constriction diameters normalized by $\mathrm{D}_{0 \mathrm{~F}}$ tend to increase with $\mathrm{C}_{\mathrm{u}}$. This is logical as the average filter particle diameter will also increase. However, as $\mathrm{C}_{\mathrm{u}}$ $\geq 3$ the CSDs become similar and for $\mathrm{C}_{\mathrm{u}} \geq 4.5$ the CSDs are practically the same. Shire et al. (2015) present data which shows that small filter particles are more likely to form constrictions, and this probably accounts for the similarity of the CSDs for higher $\mathrm{C}_{\mathrm{u}}$ materials.

The CSDs normalized by the characteristic filter diameter $\mathrm{D}_{15 \mathrm{~F}}$ are presented in Figures $10(\mathrm{~b}), 11(\mathrm{~b})$ and 12(c). For each method the CSDs form a relatively narrow band of curves.

The CSDs from the Weighted Delaunay Method (Fig. 10(b)) form a narrow band for the filters with $\mathrm{C}_{\mathrm{u}} \leq 4.5\left(\mathrm{D}_{\mathrm{c} 50}\right.$ ranges from $\left.0.26-0.29 \mathrm{D}_{15 \mathrm{~F}}\right)$. The CSD of the most widely graded filter $\left(C_{u}=6\right)$ lies to the left of the more narrowly graded filters $\left(D_{c 50}=\right.$ $0.23 \mathrm{D}_{15 \mathrm{~F}}$ ).

The Contact Based Method results in CSDs curves which become less steep as $C_{u}$ increases. This is particularly noticeable for the filter with $\mathrm{C}_{\mathrm{u}}=1.2$. Overall the Contact Based Method gives a broadly similar result to the Weighted Delaunay Method, with a a range of median constriction diameters of $\mathrm{D}_{\mathrm{c} 50}=$ $0.22-0.27 \mathrm{D}_{15 \mathrm{~F}}$.

Only three filters were analysed using the Watershed Method, and the three resulting CSDs are very similar when normalized by $\mathrm{D}_{15 \mathrm{~F}}$ and each has a median constriction diameter of $\mathrm{D}_{\mathrm{c} 50} \approx 0.27 \mathrm{D}_{15 \mathrm{~F}}$. 


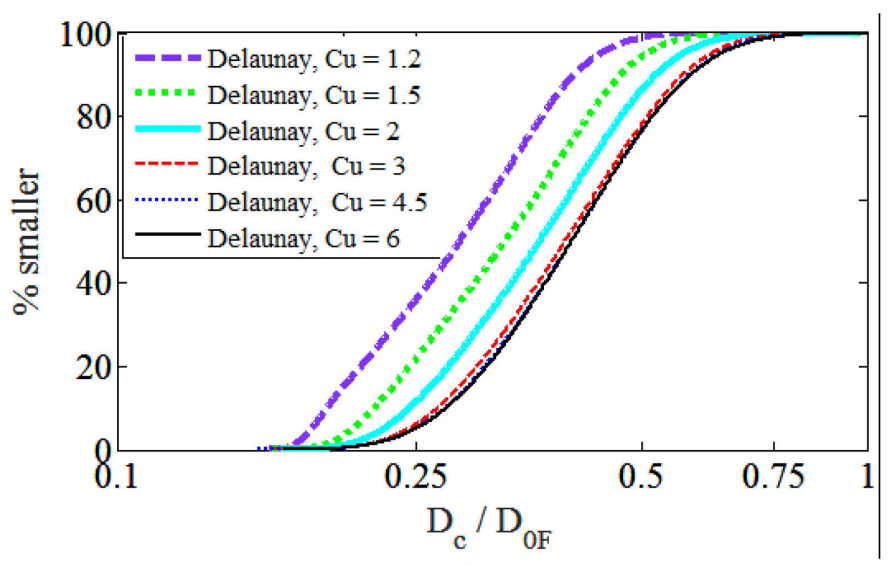

(a)

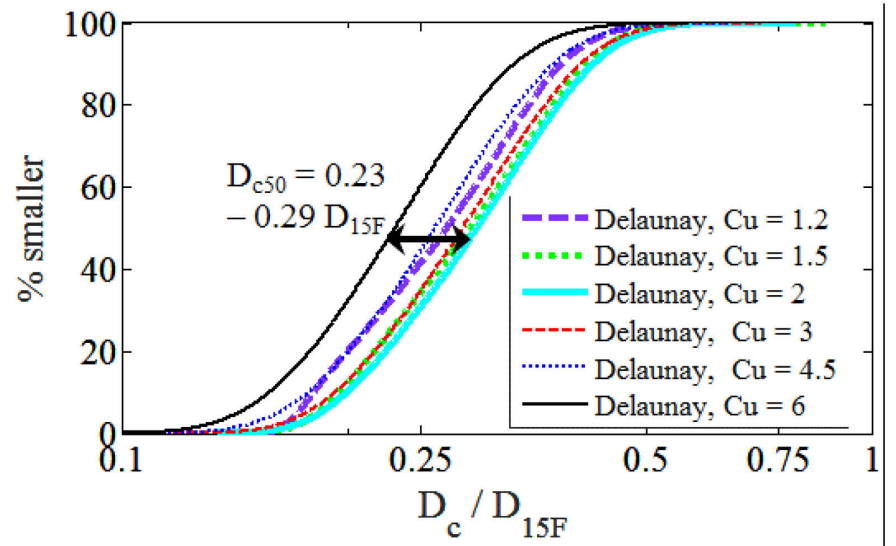

(b)

Figure 10. CSDs normalized by characteristic filter diameters for filters of $C_{u}=1.2$ to 6 from the Weighted Delaunay Method with $\mathrm{O}=50 \%$ (Shire et al., 2015).

\section{DISCUSSION AND CONCLUSIONS}

The continuous and irregular nature of void space in granular filters means that the measurement of CSDs is a subjective process. Here three methods for the measurement of CSDs are presented, each with its own logical basis. Each method results in a different CSD and in the case of the Weighted Delaunay Method different CSDs depending on the userdefined merging overlap.

Despite the difference in the methods and resulting CSDs, some common trends can be established. Firstly, for linearly graded filters with the same smallest particle $\left(\mathrm{D}_{0 \mathrm{~F}}\right)$ the constrictions become larger as the coefficient of uniformity $\left(\mathrm{C}_{\mathrm{u}}\right)$ increases from 1.2 to 3.0. However, when $\mathrm{C}_{\mathrm{u}} \geq 4.5$ the CSDs become very similar.

The second trend is that CSDs from filters in the range $C_{u}=1.2$ to 6 become relatively similar when they are normalized by the characteristic filter diameter $\mathrm{D}_{15 \mathrm{~F}}$. This agrees well with the experimental findings of Kenney et al. (1985) that the largest cohesionless base particle which could be eroded through granular filters of $\mathrm{C}_{\mathrm{u}}=1.2$ to 12 was relatively similar when normalized by $\mathrm{D}_{15 \mathrm{~F}}$. Shire et al. (2015) additionally found that CSDs differ with the relative density of the filter, but for a given relative density normalization by $\mathrm{D}_{15 \mathrm{~F}}$ or $\mathrm{D}_{5 \mathrm{~F}}$ produces similar CSDs. Together, these findings lend support to the use of $\mathrm{D}_{15 \mathrm{~F}}$ in empirical rules e.g. Terzaghi \& Peck (1948) and Sherard \& Dunnigan (1989).

\section{ACKNOWLEDGEMENTS}

The contribution of Dr. Kevin Hanley to the DEM modelling is gratefully acknowledged. The first author was funded by an EPSRC Doctoral Training Account.

\section{REFERENCES}

Al-Raoush, R., Thompson, K. \& Willson, C.S., 2003. Comparison of Network Generation Techniques for Unconsolidated Porous Media. Soil Science Society of America Journal, 67(6), p.1687.

CGAL, Computational Geometry Algorithms Library. (2013). Available at: https://www.cgal.org/.

Fannin, J., 2008. Karl Terzaghi: From Theory to Practice in Geotechnical Filter Design. Journal of Geotechnical and Geoenvironmental Engineering, 134(3), pp.267-276.

ICOLD, 2015. Bulletin on internal erosion of dams, dikes and their foundations: Volume 1., Paris.

Kenney, T.C. et al., 1985. Controlling constriction sizes of granular filters. Canadian Geotechnical Journal, 22(1), pp.32-43.

Ketcham, R. \& Carlson, W., 2001. Acquisition, optimization and interpretation of X-ray computed tomographic imagery: applications to the geosciences. Computers \& Geosciences.

Li, X. \& Li, X.-S., 2009. Micro-Macro Quantification of the Internal Structure of Granular Materials. Journal of Engineering Mechanics, 135(7), pp.641-656.

O'Sullivan, C. et al., 2015. Contact based void partitioning to assess filtration properties in DEM simulations. Computers and Geotechnics, 64, pp.120-131.

O'Sullivan, C., 2011. Particulate Discrete Element Modelling: A Geomechanics Perspective, Taylor \& Francis.

Plimpton, S., 1995. Fast Parallel Algorithms for Short-Range Molecular-Dynamics. Journal of Computational Physics, 117(1), pp.1-19.

Reboul, N., Vincens, E. \& Cambou, B., 2010. A computational procedure to assess the distribution of constriction sizes for an assembly of spheres. Computers and Geotechnics, 37(12), pp.195-206.

Sherard, J.L. \& Dunnigan, L.P., 1989. Critical Filters for Impervious Soils. Journal of Geotechnical Engineering, 115(7), pp.927-947.

Shire, T. \& O'Sullivan, C., 2015. Constriction size distributions of granular filters: a numerical study. Géotechnique, Accepted .

Taylor, H.F., O'Sullivan, C. \& Sim, W.W., 2015. A new method to identify void constrictions in micro-CT images of sand. Computers and Geotechnics, 69, pp.279-290.

Terzaghi, K. \& Peck, R.., 1948. Soil mechanics in engineering practice. Wiley 


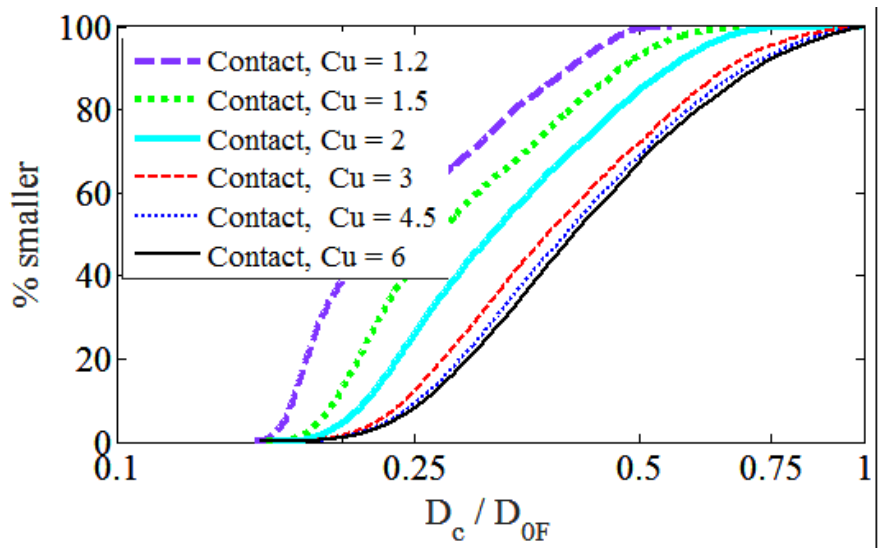

(a)

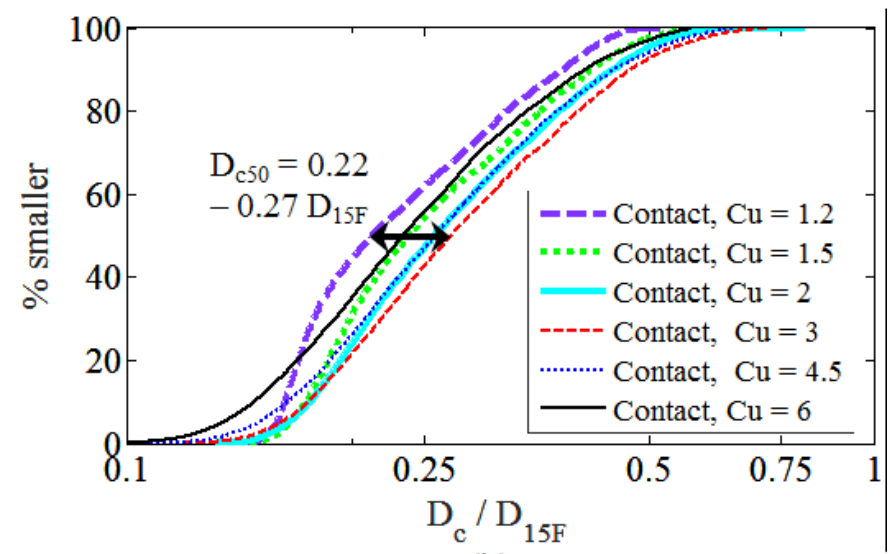

(b)

Figure 11. CSDs normalized by characteristic filter diameters for filters of $\mathrm{C}_{\mathrm{u}}=1.2$ to 6 using the Contact Method

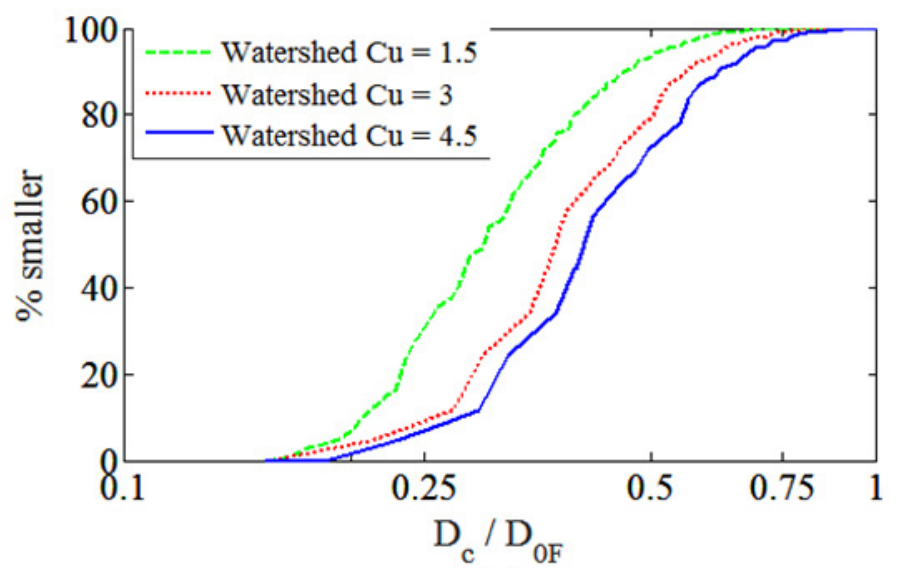

(a)

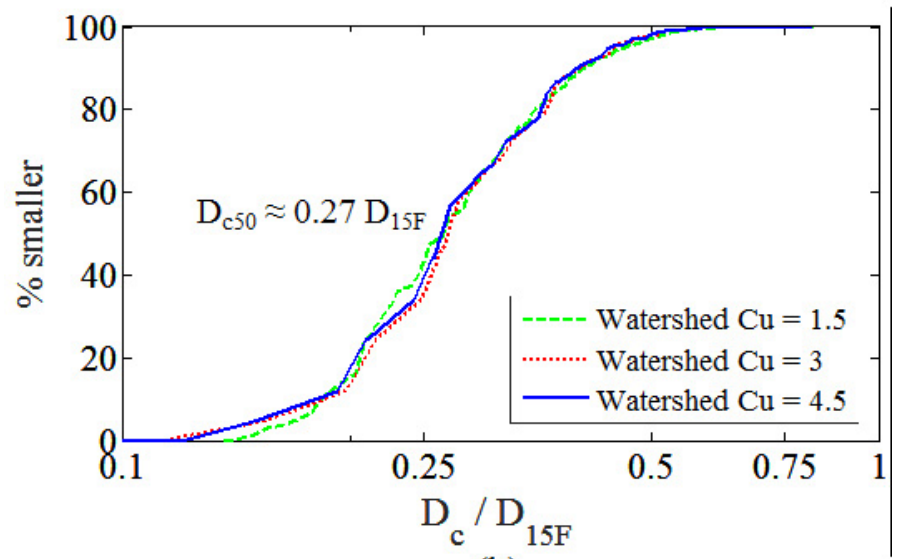

(b)

Figure 12. CSDs normalized by characteristic filter diameters for filters of $\mathrm{C}_{\mathrm{u}}=1.5$ to 4.5 using the Watershed Method 